Draft VERSION OCTOBER 27, 2020

Typeset using $\mathrm{LAT}_{\mathrm{E}} \mathrm{X}$ twocolumn style in AASTeX63

\title{
The MKID Exoplanet Camera for Subaru SCExAO
}

\author{
Alexander B. Walter, ${ }^{1}$ Neelay Fruitwala, ${ }^{2}$ Sarah Steiger (D), ${ }^{2}$ John I. Bailey, III (D),${ }^{2}$ Nicholas Zobrist, ${ }^{2}$ \\ Noah Swimmer D,$^{2}$ Isabel Lipartito (D), ${ }^{2}$ Jennifer Pearl Smith ${ }^{D},{ }^{2}$ Seth R. Meeker, ${ }^{1}$ Clint Bockstiegel, ${ }^{2}$ \\ Gregoire Coiffard ${ }^{2}$ Rupert Dodkins, ${ }^{2}$ Paul Szypryt, ${ }^{3,4}$ Kristina K. Davis (iD, ${ }^{2}$ Miguel Daal, ${ }^{2}$ Bruce Bumble, ${ }^{1}$ \\ Giulia Collura, ${ }^{2}$ Olivier Guyon, ${ }^{5}$ Julien Lozi, ${ }^{5}$ Sebastien Vievard, ${ }^{5}$ Nemanja Jovanovic (iD, 6 \\ Frantz Martinache, ${ }^{7}$ Thayne Currie, ${ }^{5,8,9}$ And Benjamin A. Mazin (iD) 2 \\ ${ }^{1}$ Jet Propulsion Laboratory, California Institute of Technology, Pasadena, California 91125, USA \\ ${ }^{2}$ Department of Physics, University of California, Santa Barbara, CA 93111, USA \\ ${ }^{3}$ National Institute of Standards and Technology, Boulder, Colorado 80305, USA \\ ${ }^{4}$ Department of Physics, University of Colorado, Boulder, Colorado 80309, USA \\ ${ }^{5}$ National Astronomical Observatory of Japan, Subaru Telescope, Hilo, HI 96720 \\ ${ }^{6}$ Caltech Optical Observatories, California Institute of Technology, Pasadena, CA 91125 \\ ${ }^{7}$ Université Côte d'Azur, Observatoire de la Côte d'Azur, CNRS, Laboratoire Lagrange, France \\ ${ }^{8}$ NASA-Ames Research Center, Moffett Blvd., Moffett Field, CA, USA \\ ${ }^{9}$ Eureka Scientific, 2452 Delmer Street Suite 100, Oakland, CA, USA
}

\begin{abstract}
We present the MKID Exoplanet Camera (MEC), a $z$ through J band (800-1400 nm) integral field spectrograph located behind The Subaru Coronagraphic Extreme Adaptive Optics (SCExAO) at the Subaru Telescope on Maunakea that utilizes Microwave Kinetic Inductance Detectors (MKIDs) as the enabling technology for high contrast imaging. MEC is the first permanently deployed near-infrared MKID instrument and is designed to operate both as an IFU, and as a focal plane wavefront sensor in a multi-kHz feedback loop with SCExAO. The read noise free, fast time domain information attainable by MKIDs allows for the direct probing of fast speckle fluctuations that currently limit the performance of most high contrast imaging systems on the ground and will help MEC achieve its ultimate goal of reaching contrasts of $10^{-7}$ at $2 \lambda / D$. Here we outline the instrument details of MEC including the hardware, firmware, and data reduction and analysis pipeline. We then discuss MEC's current on-sky performance and end with future upgrades and plans.
\end{abstract}

Keywords: MKIDs — OIR Instruments — Direct Imaging — Adaptive Optics

\section{INTRODUCTION}

The discovery and characterization of exoplanets via direct imaging is particularly challenging due to the extreme contrasts $\left(<10^{-4}\right.$ for ground based targets) and small angular separations $\left(\lesssim 1^{\prime \prime}\right)$ between the planetary companion and it's host star. For a review of the direct imaging of giant planets over the past few decades, see Bowler (2016). Sophisticated ground-based high contrast instruments have been, or are being, built including: the Gemini Planet Imager (GPI, Macintosh et al. 2008, 2014), SPHERE at VLT (Carbillet et al. 2011; Beuzit et al. 2019), SCExAO at Subaru (Jovanovic et al. 2015), P1640 (Crepp et al. 2011; Lewis \& Oppenheimer 2017) and the Stellar Double Coronagraph (SDC, Mawet

Corresponding author: Sarah Steiger

steiger@ucsb.edu et al. 2014a) at Palomar, the Keck Planet Imager and Characterizer (KPIC) at Keck (Mawet et al. 2016; Jovanovic et al. 2019), and MagAO-X at the Magellan Clay Telescope (Males et al. 2018). Adaptive optics (AO) and coronagraphy have enabled the discovery of planets with contrasts down to $\sim 10^{-6}$. This has allowed researchers to survey formation conditions in primordial disks as well as the temperature of a handful of giant young exoplanets in the stellar neighborhood (Marois et al. 2008; Lagrange et al. 2010; Kuzuhara et al. 2013; Currie et al. 2014; Macintosh et al. 2015; Keppler et al. 2018).

High-contrast imaging is limited primarily by uncontrolled diffracted light which produces a coherent speckle halo in the image plane. Speckles can arise from atmospheric aberrations, or from the non-ideal nature of the instrument, the latter suffering from noisy near-infrared (IR) detectors, time lag in the AO correction, speed of the AO control loop, non-common path errors, telescope vibrations, and chromaticity between the wavefront and 
science cameras (Guyon 2005; Lozi et al. 2018). Atmospheric speckles are fast (rapidly evolving) and average down over an observation, while the slower, quasi-static speckles resulting from the non-ideal nature of the instrument must be removed using post-processing techniques. Angular differential imaging (ADI, Marois et al. 2006) exploits the rotation of the Earth or, analogously, the field-of-view of an altitude-azimuth telescope, to distinguish diffraction speckles, which remain stationary, from astrophysical sources, which will rotate with the frame. Spectral differential imaging (SDI, Racine et al. 1999; Marois et al. 2000; Sparks \& Ford 2002) uses the scaling of diffraction speckles with wavelength to distinguish speckles from true astrophysical sources. Since the initial development of ADI and SDI, a variety of post-processing algorithms have refined their approaches to push for higher achievable contrasts (e.g. Lafrenière et al. 2007; Soummer et al. 2012; Marois et al. 2014; Gomez Gonzalez, C. A. et al. 2016).

The time variability and chromaticity of quasi-static speckles, however, limit the performance of ADI and SDI which are the best performing current techniques (Gerard et al. 2019). Both of these techniques also suffer at small angular separations where exoplanets are more likely to occur. The speckle spectral dispersion used by SDI and the arclength traced by the companion's sky rotation used by ADI, are both proportional to the planet's separation. Furthermore, the precision of the background estimate for PSF subtraction is limited by low counting statistics at small separations (Mawet et al. 2014b). Even without these issues, the variability induced by speckle fluctuations can dominate over the shot noise expected from the total number of photons.

A significant improvement over existing systems can be achieved by using read-noise free time domain information on short enough timescales to sample and control, or remove in post-processing, the fast speckle fluctuations from imperfections in the $\mathrm{AO}$ system and non-common path aberrations. We have constructed the MKID Exoplanet Camera (MEC) for Subaru Telescope's SCExAO system for this purpose - to serve as both a photon counting, energy resolving science camera, and as a focal plane wavefront sensor to correct for chromatic wavefront errors not accounted for by the upstream AO system. To achieve this, a high-speed, noise-free detector is required, like the Microwave Kinetic Inductance Detector (MKID) array used by MEC $(\S 2.1)$.

This instrument paper will highlight MEC's capabilities, the performance of the combined MEC and SCExAO system for high contrast imaging of exoplanets and disks, and document the significant new technology developed for MEC.

\section{MEC SYSTEM DESCRIPTION}

MEC was commissioned at Subaru Observatory in 2018 and is designed as a $z$ through J-band IFU for

\begin{tabular}{cc}
\hline \hline Parameters & Values \\
\hline Device Materials & PtSi on Sapphire w/ Nb g.p. \\
Device Format & 140x146 pixels (10 feedlines $)$ \\
Pixel Pitch & $150 \mu \mathrm{m}$ \\
Plate Scale & $10.4 \mathrm{mas} / \mathrm{pixel}$ \\
Field of View & $1.4 " \mathrm{x} 1.5 "$ \\
Wavelength Band & $800-1400 \mathrm{~nm}(z-\mathrm{J})$ \\
Spectral Resolution $(\lambda / \Delta \lambda)$ & $5-7$ \\
Max Count Rate & $5000 \mathrm{cts} / \mathrm{pix} / \mathrm{second}$ \\
Pixel Dead Time & $10 \mu \mathrm{s}$ \\
Readout Frame Rate & $>2 \mathrm{kHz}$ \\
Operating Temp. & $90 \mathrm{mK}$ \\
4 K Stage Base Temp. & $3.1 \mathrm{~K}$ \\
60 K Stage Base Temp. & $57 \mathrm{~K}$ \\
90 mK Hold Time & $>17 \mathrm{hours}$ \\
\hline
\end{tabular}

Table 1. MEC Instrument Summary

high contrast imaging and as a focal plane wavefront sensor for a multi-kHz feedback loop with SCExAO. The enabling technology are Microwave Kinetic Inductance Detectors (MKIDs), which are capable of color discriminating near-IR photons with read noise free microsecond photon timing. The unique capabilities of MKIDs, coupled with SCExAO, provide a powerful means of overcoming the limits of ground based high contrast imaging and acts as a testbed for technologies aimed at future $30 \mathrm{~m}$ class telescopes.

MEC's requirements were based on the delivered optical beam from SCExAO, the available space to mount MEC to SCExAO, the performance of the MKIDs, and the available budget. This resulted in a design with the instrument parameters that are summarized in Table 1.

\subsection{Optical and Infrared (OIR) MKID Arrays}

MKIDs work on the principle that incident photons change the surface impedance of a superconductor through the kinetic inductance effect. The kinetic inductance effect occurs because energy can be stored in the supercurrent (the flow of Cooper Pairs) of a superconductor. Reversing the direction of the supercurrent requires extracting the kinetic energy stored in it, which yields an extra inductance term in addition to the familiar geometric inductance. The magnitude of the change in surface impedance depends on the number of Cooper Pairs broken by incident photons, and hence is proportional to the amount of energy deposited in the superconductor. This change can be accurately measured by placing a superconducting inductor in a lithographed resonator. A microwave probe signal is tuned to the resonant frequency of the resonator and any photons which are absorbed in the inductor will imprint their signature as changes in the phase and amplitude of this probe signal. 

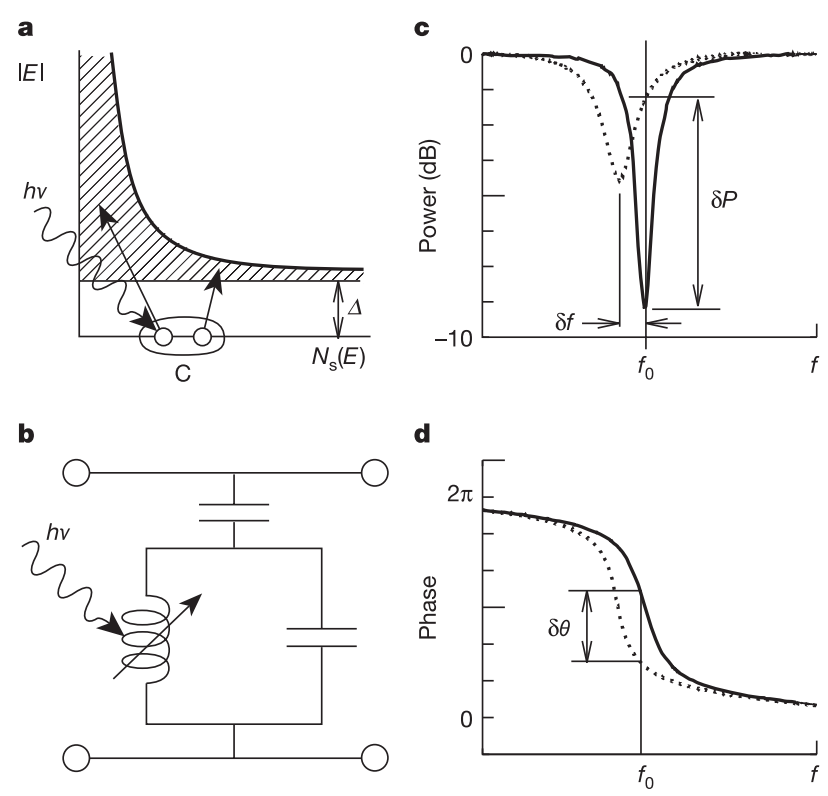

Figure 1. The basic operation of an MKID, from Day et al. (2003). (a) Photons with energy $h \nu$ are absorbed in a superconducting film producing a number of excitations called quasiparticles. (b) To sensitively measure these quasiparticles the film is placed in a high frequency planar resonant circuit. The amplitude (c) and phase (d) of a microwave excitation signal sent through the resonator. The change in the surface impedance of the film following a photon absorption event pushes the resonance to lower frequency and changes its amplitude. If the detector (resonator) is excited with a constant on-resonance microwave signal, the energy of the absorbed photon can be determined by measuring the degree of phase and amplitude shift.

Since the quality factor, Q, of the resonators is high, and their transmission off resonance is nearly perfect, multiplexing can be accomplished by tuning each pixel to a different resonant frequency with lithography during device fabrication. A comb of probe signals is sent into the device and room temperature electronics recover the changes in amplitude and phase. This makes a device capable of measuring the arrival time (to a microsecond) and energy (to 5-10\%) of each arriving photon without read noise or dark current; an optical/nearIR analog of an X-ray microcalorimeter. More details on MKIDs can be found in Mazin et al. $(2012,2013)$ and in Figure 1.

The MEC MKID array has 10 coplanar waveguide (CPW) transmission lines, or feedlines, that each probe 14 x 146 pixels for a total of 20440 pixels in the array. This makes it the largest superconducting detector in the world. It uses the PtSi MKIDs described in Szypryt et al. (2017), which are optimized for 800-1400 nm light with a spectral resolution of about $\lambda / \Delta \lambda=6$. The pix-

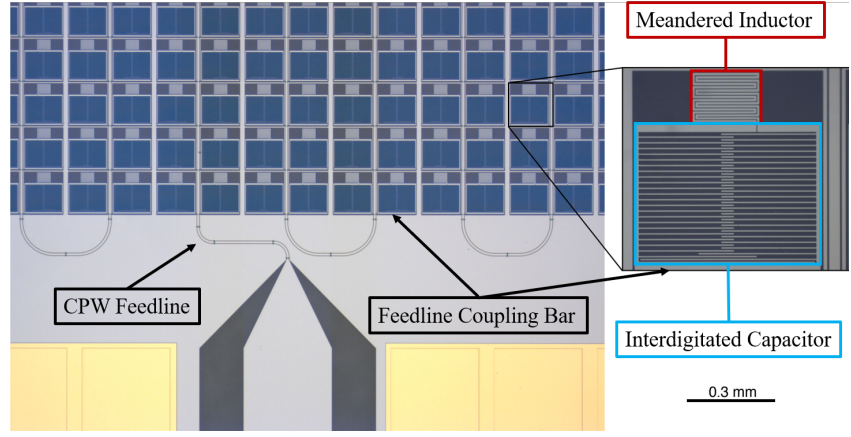

Figure 2. Microscope image of a small section of a $20 \mathrm{kpix}$ MKID array similar to the engineering array in the MEC instrument. Left: A coplanar waveguide (CPW) feedline with crossovers connects to a grid of MKID pixels. Gold thermal pads are shown at the bottom. Right: Zoomed in picture showing the large interdigitated capacitor, meandered inductor, and the feedline coupling bar (bottom right corner).

els are on a $150 \mu m$ pitch, see Figure 2. The device currently in MEC has 7/10 feed lines with good transmission and on those there is about an $80 \%$ pixel yield. More detail on current performance can be found in $\S 3$.

\subsection{Cryostat}

MEC's cryostat is a pulse tube cooled Adiabatic Demagnetization Refrigerator (ADR) capable of reaching temperatures below $50 \mathrm{mK}$. The outer vacuum shell $300 \mathrm{~K}$ enclosure measures roughly $22 \mathrm{~cm}$ wide $\mathrm{x} 33 \mathrm{~cm}$ deep x $96 \mathrm{~cm}$ tall. It contains $\mathrm{RF}$ gaskets to reduce radio frequency interference, a $7.5 \mathrm{~kW}$ pulse tube, and all the vacuum and wiring connections to accommodate a 20 kpix MKID array.

The $60 \mathrm{~K}$ shell is cooled by the first stage of the pulse tube. It contains heat sinks to cool the wiring to reduce heat load on the $4 \mathrm{~K}$ stage. The $4 \mathrm{~K}$ stage is cooled by the second stage of the pulse tube, and has $0.75 \mathrm{~W}$ of cooling power at $4 \mathrm{~K}$.

Attached to the $4 \mathrm{~K}$ plate, shown in Figure 3 are: the ADR unit with mechanical heat-switch, the ten cryogenic high-electron-mobility transistor (HEMT) amplifiers, and the detector package. The detector package is mounted to the $4 \mathrm{~K}$ plate and is enclosed in a $4 \mathrm{~K}$ magnetic shield. This design places the MKID array far from the magnetic shield opening where field leakage will be strongest and also allows us to install a $1 \mathrm{~K}, 9 \mathrm{~cm}$ long black baffle to further reduce off-axis scattered light and $4 \mathrm{~K}$ black body radiation. The MKID array is mounted in a microwave package $5 \mathrm{~cm} \times 5 \mathrm{~cm} \times 0.5 \mathrm{~cm}$ in size, as shown in Figure 4. This box is attached to a gold-plated copper rod that sticks out of the base of the magnetic shield and connects to the ADR unit by a copper strap.

The ADR acts as a single-shot magnetic cooler which brings the MKID array down to $90 \mathrm{mK}$ where the temperature is stabilized with a feedback loop to the ADR magnet power supply. We achieve a $90 \mathrm{mK}$ hold time 


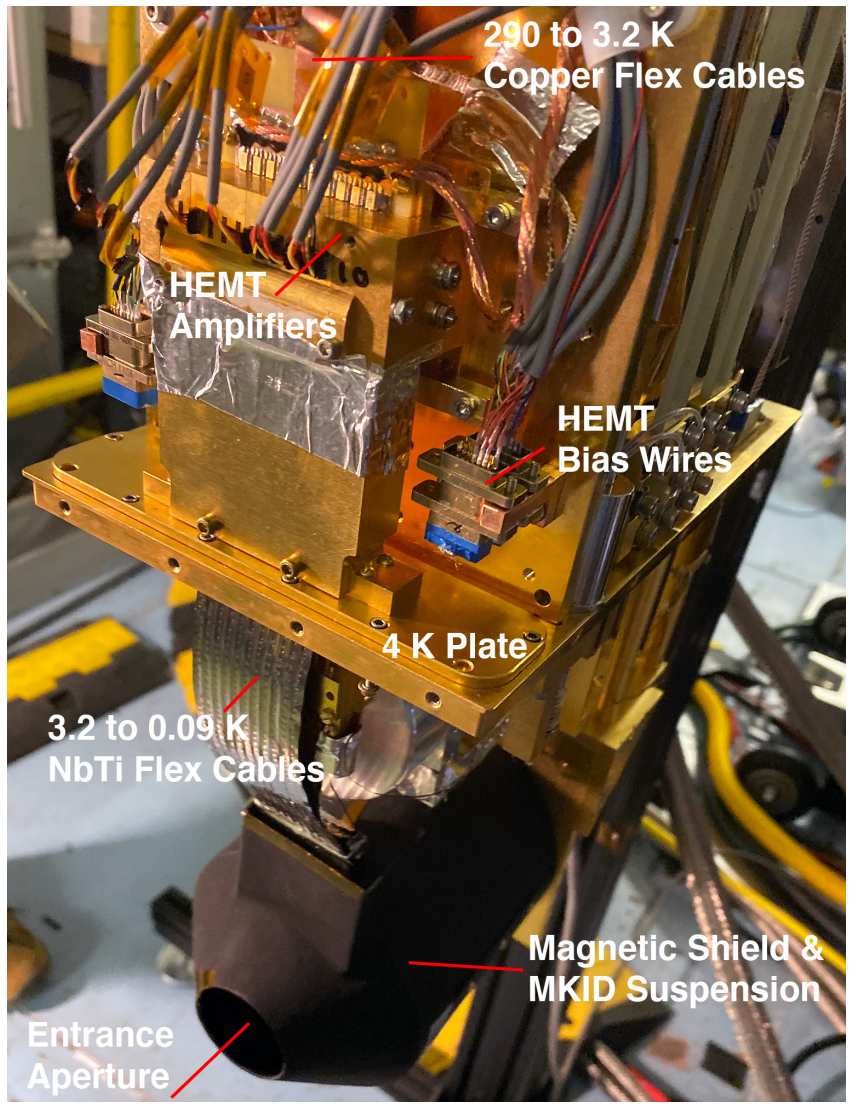

Figure 3. Image of the $4 \mathrm{~K}$ stage of $\mathrm{MEC}$ with the radiation shields removed to show the readout wiring and the black magnetic shield. The pulse tube and heat switch are hidden by the wiring attachment plate.

of $>17$ hours on the telescope, more than sufficient for the 13-14 hours required for a night of calibration and on-sky observations.

\subsection{Readout}

\subsubsection{Readout Electronics}

The electronics rack (E-rack) is situated on the Nasmyth platform next to the MEC instrument (Figure 5). It requires $3.5 \mathrm{~kW} \max$ of power supplied to the power distribution unit with a $30 \mathrm{~A}, 208 \mathrm{~V}$ 3-phase power cord. Cooling water/glycol is supplied to the E-rack by Subaru. There should be unobstructed air flow to two readout crates in the electronics rack. The front (facing SCExAO) draws air in, and the back expels air through a heat exchanger into the Nasmyth room.

The readout for MEC is based on the ARCONS readout, which is detailed in McHugh et al. (2012) and is identical to the DARKNESS readout detailed in Strader (2016); Fruitwala (Submitted). The readout system is responsible for generating the RF frequency comb to drive the resonators, and for digitizing and processing the resulting MKID array output to determine the ar-

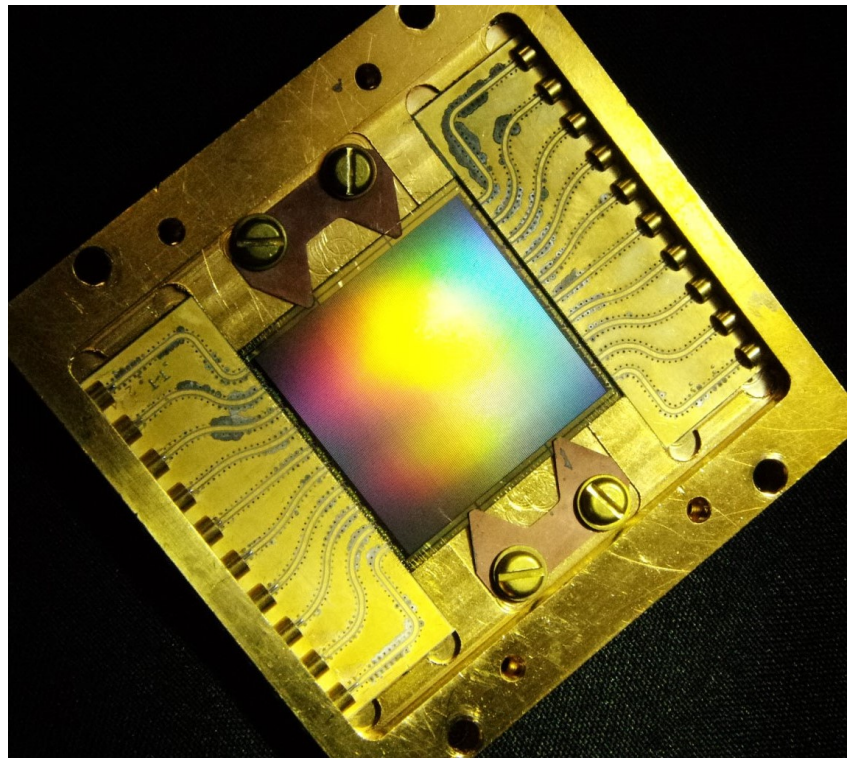

Figure 4. Image of a 20440 pixel PtSi MKID device designed for MEC. This is the highest pixel count superconducting detector array at any wavelength.

rival time energy of any incident photons, see §2.3.2. The MEC readout employs twenty second generation CASPER Reconfigurable Open Architecture Computing Hardware (ROACH2) boards. Each of these ROACH2 boards are connected to an Analog to Digital/Digital to Analog Converter (ADC/DAC) board and a Radio Frequency/Intermediate Frequency (RF/IF) board, both of which are designed at Fermilab (See Figure 6 for one board set). Each set of boards reads out 1024 pixels in $2 \mathrm{GHz}$ of bandwidth.

Every ROACH2 houses a Xilinx Virtex6 Field Programmable Gate Array (FPGA), which processes and channelizes the signal from the ADC using a scaled up version of the ARCONS firmware. The ADC/DAC board houses a Virtex7 FPGA. The ROACH2 and ADC/DAC boards are connected by two Z-Dok connectors, which are responsible for sending the ADC ouptut and clock signals to the ROACH2, and for implementing an SPI communications interface between the Virtex- 6 and Virtex-7 FPGAs.

The ROACH2 boards are on a private local-area network (LAN) as moderated by the network switch. We run all readout control software on the MEC data server, however, any computer (with the required Python libraries) that is connected to the LAN can perform this task. We use a PPS (pulse per second) signal generator to sync the clocks for each set of readout boards. The PPS signal is sychronized to an external PTP/NTP server to ensure accurate absolute timing as well as synchronization with the data server clock (See Figure 7).

\subsubsection{Readout Procedure}




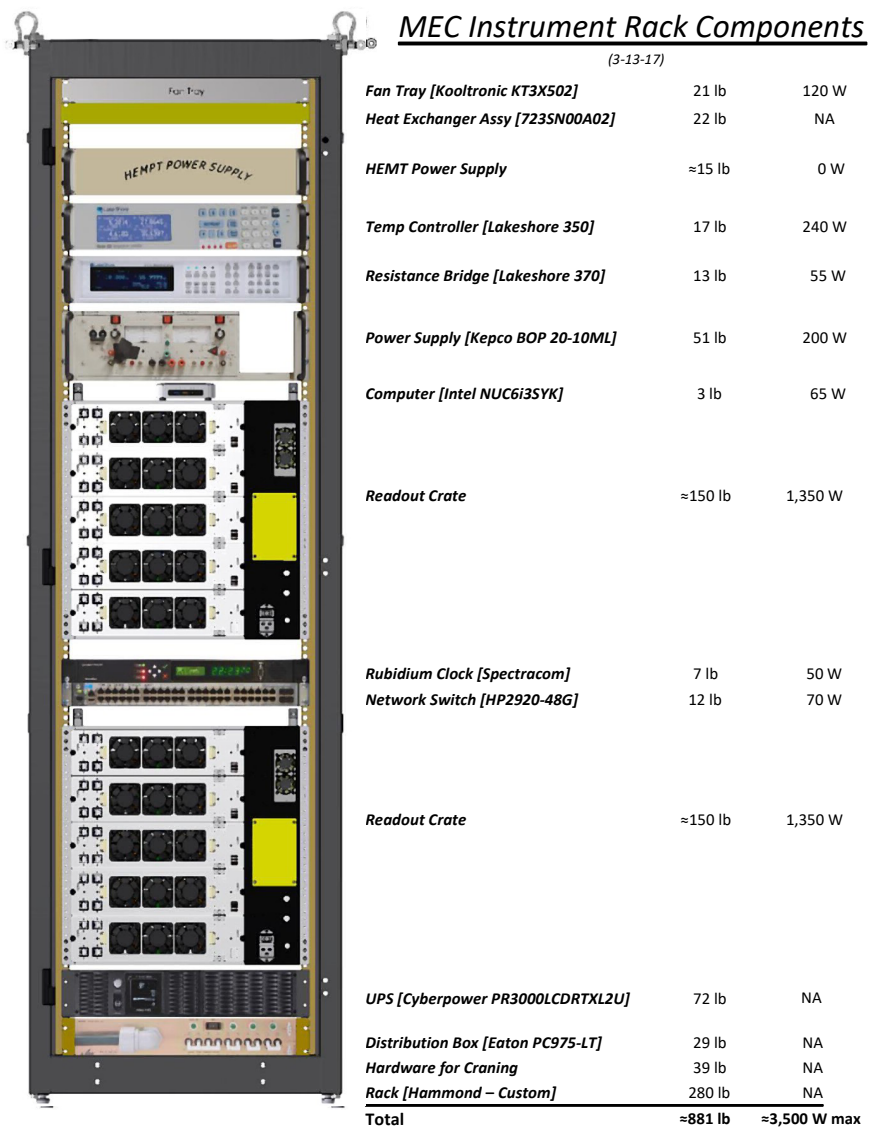

Figure 5. CAD drawing of MEC electronics rack (E-rack) and list of components.

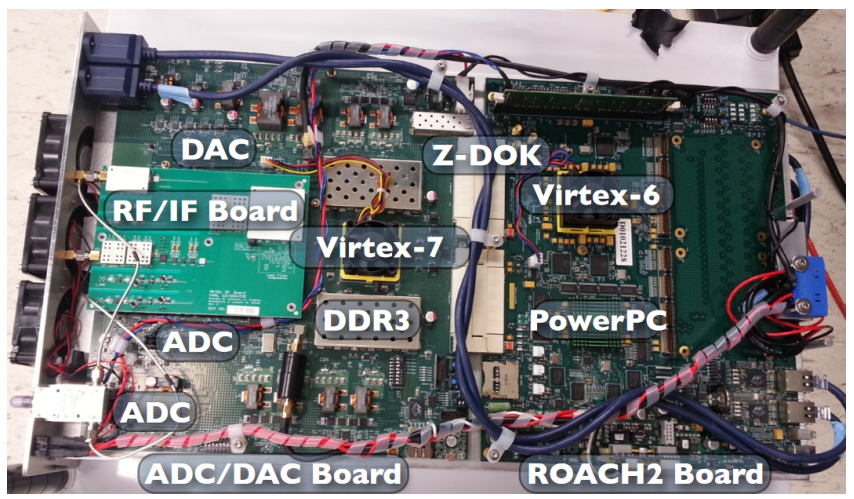

Figure 6. The ROACH2 board is connected to the $\mathrm{ADC} / \mathrm{DAC}$ board by two Z-DOK connectors. The RF/IF board is mounted on the ADC/DAC board using SMP blind-mate connectors for signals and general-purpose input/output (GPIO) pins for programming. Another set of three boards are mounted to the underside of this cartridge. Figure and Caption reproduced from Strader (2016).

The initial setup defines the frequency comb that probes the resonators. This is determined by doing frequency sweeps of every feedline at a grid of powers and then using a machine learning code to both find the resonators and determine their optimal probe powers. An algorithm to find the probe power of known resonators is outlined in Dodkins et al. (2018), but has been superseded by an algorithm that simultaneously finds resonances and their ideal power which will be detailed in an upcoming publication. Our python software then computes a lookup table (LUT) containing the time-domain sum of the resonator probe tones. The lookup table is stored in DDR3 RAM onboard the ADC/DAC board, and is played to the DAC in a loop while the system runs. The DAC output is passed through the $\mathrm{RF} / \mathrm{IF}$ board where an IQ mixer upconverts the signal to the right frequency range ( 4 to $6 \mathrm{GHz}$ or 6 to $8 \mathrm{GHz}$ ) using a local oscillator. After traveling through the cryostat, MKID array, and HEMTs, the RF/IF board mixes down the returning signal to baseband where it is digitized by the ADCs. Then the Virtex7 on the ADC/DAC board streams the raw ADC output to the ROACH2 over the ZDOK connectors. The ROACH2 firmware processes ADC data stream, performing the following actions:

1. Separate the comb of frequencies into individual pixel frequencies using an FFT (Fast Fourier Transform) and digital down conversion (DDC). 


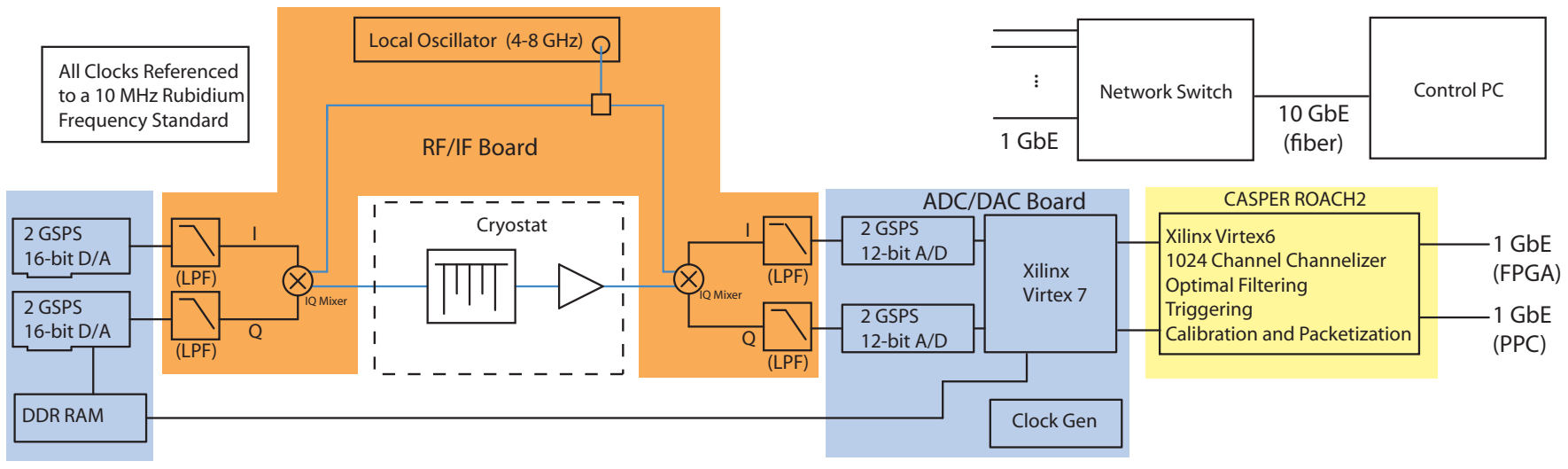

Figure 7. Readout system block diagram. This diagram is for 1 set of boards which can read out 1024 pixels at a time. Figure reproduced from Meeker et al. (2018a)

2. Sample the phase of each resonator's signal every microsecond.

3. Run a pixel-wise unique optimal filter over each pixel's phase signal.

4. Trigger on photon events and store photon packets in buffer.

5. Send buffer to disk every 0.5 millisecond (or faster if full) over 1-Gbit Ethernet via UDP.

Each single photon packet is a 64 bit word with the following breakdown: 10 bit $\mathrm{x}$-coordinate, 10 bit $\mathrm{y}$ coordinate, 9 bit timestamp, 18 bit wavelength, 17 bit baseline phase. A C program on the data server receives the UDP frames containing photon packets sent from the ROACH2 boards and writes them to disk as observation files. Additionally, it histograms the photons into images for real time display.

\subsection{Optics}

\subsubsection{Specifications of Incoming Beam}

The SCExAO exit beam is located $293 \pm 1 \mathrm{~mm}$ from the top of the top mounting rail. The beam comes off the bench at about $58 \mathrm{~mm}$ from the left edge of the bench. The SCExAO exit beam is collimated. The pupil position is $54 \pm 20 \mathrm{~mm}$ from the back of the bench. The pupil size is $8.67 \pm 0.15 \mathrm{~mm}$. The beam is expected to exhibit $<10 \mathrm{~nm}$ of chromatic RMS optical wavefront error over the 0.8-1.4 $\mu \mathrm{m}$ bandwidth.

\subsubsection{MEC Fore-Optics}

The MEC optics box has a two inch hole for the incoming SCExAO beam (Figure 8). A set of three lenses creates a telecentric beam designed to reimage the focus onto the MKID array as simulated in Zemax. The beam leaves the optics box with an f-number $f / 377.7$, travels through the neutral density (ND) filter wheel (Table 3), and enters the cryostat through the front vacuum window. There are two IR bandpass filters at the $60 \mathrm{~K}$ and

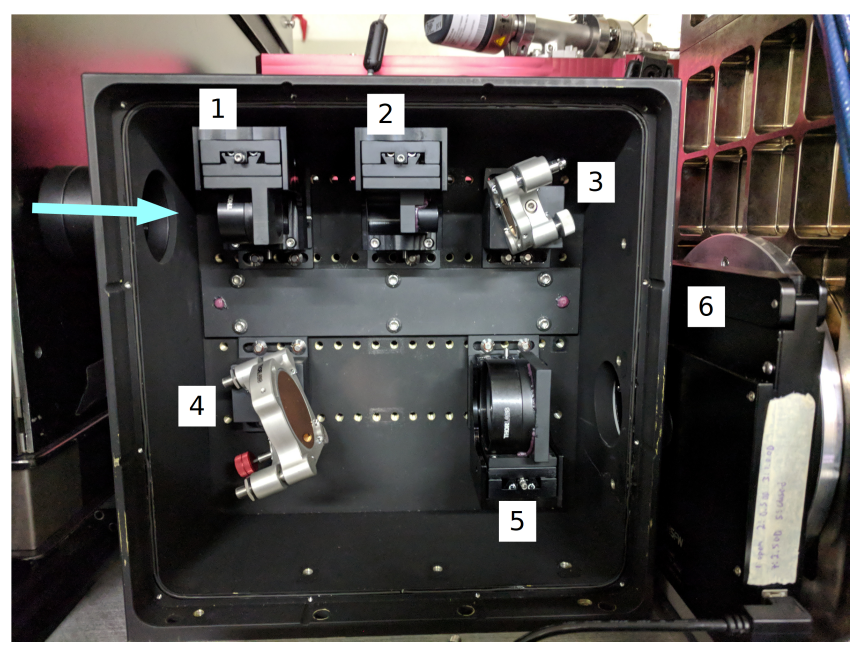

Figure 8. The MEC foreoptics box reimages the beam from SCExAO (arrow from left) onto the MKID device. Two gold mirrors (3 and 4) and three lenses (1,2 and 5) steer and focus the input collimated beam onto the MKID array with an f-number of $\mathrm{f} / 377.7$. The specific optics components can be found with their corresponding numbers in Table 2.

$4 \mathrm{~K}$ shield to block black body radiation and finally a microlens array focuses the beam onto the light sensitive part of the MKID pixels. The MEC optics are listed in Table 2.

\subsection{Integration with SCExAO}

As of March 19, 2018, MEC is mounted on the Nasmyth platform behind SCExAO in the place of the High-Contrast Coronographic Imager for Adaptive Optics (HiCIAO; Hodapp et al. (2008)). The E-rack is stationed next to MEC on the Nasmyth floor. A He compressor is located in the IR compressor room on the observation floor and a linux server is in the server room on the first floor of the control building. Figure 10 diagrams how MEC is situated on the Nasmyth floor. Figure 9 is an image of MEC mounted at the telescope. 
Table 2. MEC Optics

\begin{tabular}{cll}
\hline \hline$\#$ & Optic & Description \\
\hline 1 & $100 \mathrm{~mm}$ FL lens & Thorlabs AC254-100-c \\
2 & $9 \mathrm{~mm}$ FL lens & Edmunds 45-783 \\
3 & $2.54 \mathrm{~mm}$ gold mirror & Mnt. on CONEX-AG-M100D \\
4 & $5.08 \mathrm{~mm}$ gold mirror & Fixed mount \\
5 & $300 \mathrm{~mm}$ FL lens & Thorlabs AC508-300-c \\
6 & 2 in ND Filter wheel & See Table 3 \\
7 & Front Window & Edmund 48-130 \\
8 & $60 \mathrm{~K} \mathrm{IR} \mathrm{filter}$ & 10 mm thick N-BK7 \\
9 & 4 K IR filter & 20 mm thick N-BK7 \\
10 & Microlens Array & a $\mu$ s APO-GT-P150-R0.8 \\
\hline
\end{tabular}

Table 3. MEC Neutral Density Filter Wheel

\begin{tabular}{ccl}
\hline \hline Slot & Filter & Part \# \\
\hline 1 & OPEN & \\
2 & 0.5 OD & Edmund 62-673 \\
3 & 1.0 OD & Edmund 62-676 \\
4 & 2.5 OD & Edmund 62-680 \\
5 & CLOSED & \\
\hline
\end{tabular}

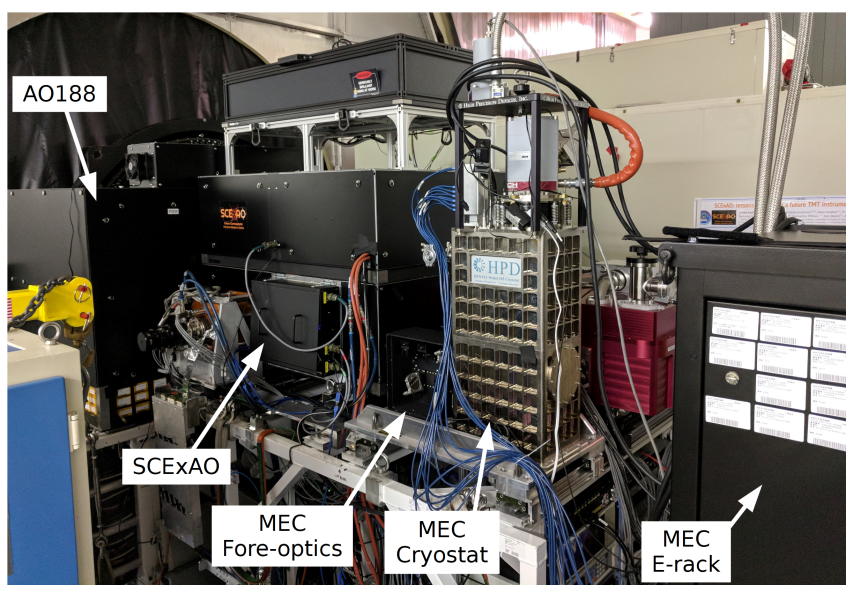

Figure 9. MEC is mounted behind the SCExAO bench and AO188 facility adaptive optics systems on the IR Nasmyth port at Subaru Telescope.

\subsection{Data Processing/Calibration}

To analyze MEC data we use The MKID Pipeline available on Github: https://github.com/MazinLab/ MKIDPipeline.

The MKID Pipeline begins by transforming the raw binary data into calibrated photon lists in a fast access database. This process is outlined in Figure 11. Many of the steps were implemented for the ARCONS pipeline

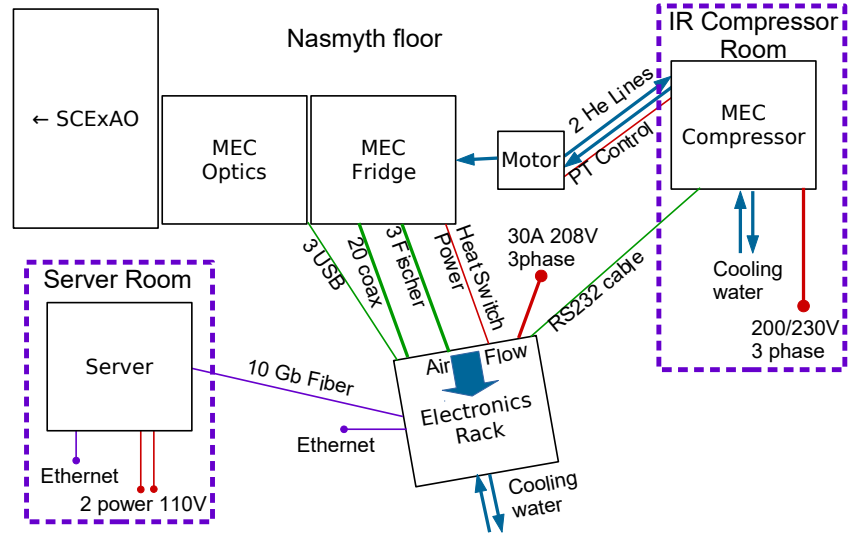

Figure 10. A diagram showing the MEC floor plan and interconnects at the Subaru Telescope. Here, red lines denote power connections, blue lines/arrows denote cooling lines, green lines denote electrical cabling and purple lines denote network connections.

(van Eyken et al. 2015) and were copied or slightly modified for MEC.

1. Processing Binary Data into a HDF5 Database

The raw binary observation files, consisting of header and photon data sent over ethernet, are interpreted and saved into a HDF5 table format. The table has one entry for each photon with the following fields: pixel ID, arrival time, phase height (or wavelength), and two additional weight fields to be set in later calibration steps (spectral weight and noise weight). The photon list is rearranged so that it is first ordered by pixel location and then by time.

2. Cosmic Ray Rejection

Cosmic rays incident on an MKID detector have the effect of illuminating most of the array with false high energy photon counts. This calibration step removes time chunks from further analysis that are identified as containing a cosmic ray. The advantage of MKID's timing resolution is that these time chunks can be precisely selected to remove an average of only $0.01 \%$ of a given observation. This retains as much of the original exposure time as possible.

3. Wavelength Calibration

The wavelength calibration is essential to utilize MKID's energy resolving capabilities. Several monochromatic laser exposures are used to determine a quadratic, $a^{2} \phi+b \phi+$ $c=h c / \lambda$, that converts phase, $\phi$, into wavelength, $\lambda$ for each pixel. To save space, the 


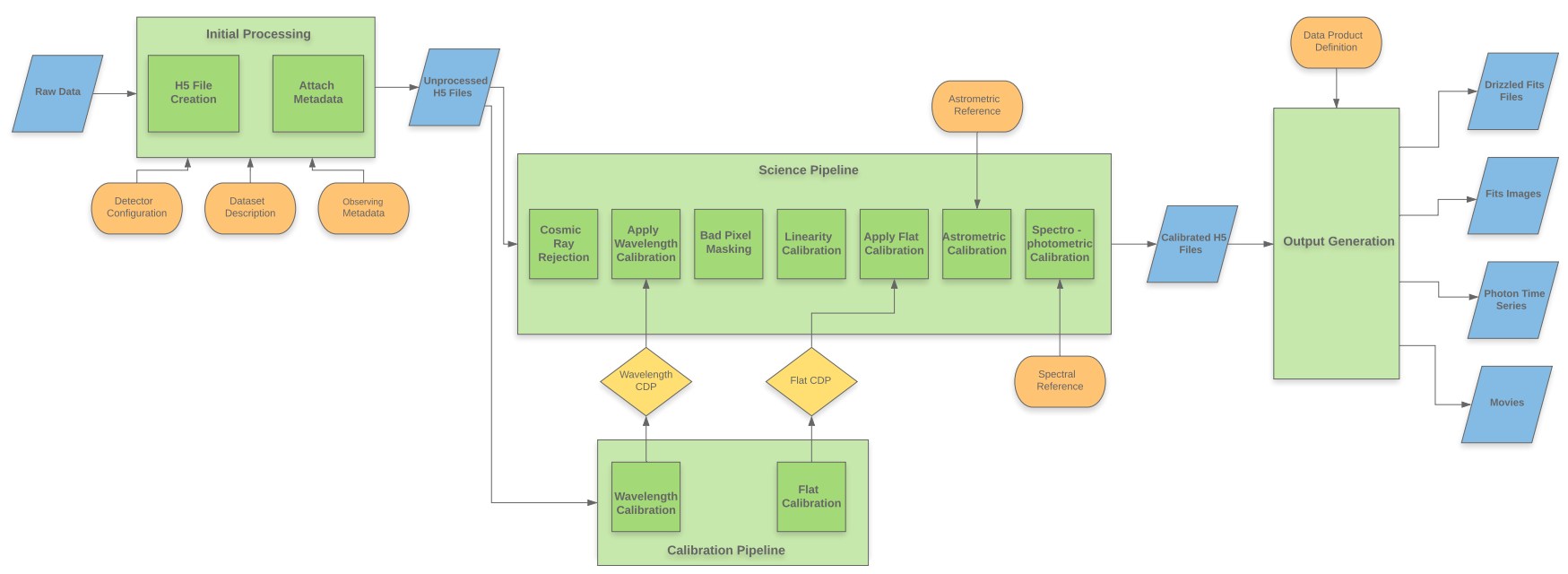

Figure 11. Block Diagram of The MKID Pipeline. Calibration steps are shown in green, references and configurations are shown in orange, data products are shown in blue, and calibration data products (CDP) are shown in yellow.

phase height float column is overwritten with the calibrated wavelength for each photon that has a valid wavelength solution.

\section{Bad Pixel Masking}

Hot, cold, or otherwise badly behaving pixels are prevalent in our data since we try to include as many pixels as possible from the readout step. These pixels are identified by determining whether their measured flux value deviates from the median flux of pixels in a surrounding box by a user specified number of standard deviations. These pixels can then be removed from the final data product.

\section{Linearity Correction}

This calibration accounts for missing photons due to the detector dead time. This dead time $(10 \mu s$ for MEC) is introduced in the firmware to avoid photon pile-up and limit the total count rate, but results in a nonlinear detector response at high count rates. To account for this effect, a correction factor is applied to the spectral weight column for each photon depending on the local count rate.

\section{Flat Field Calibration}

This is a standard flat field calibration that determines the inter-pixel relative QE. This calibration is done as a function of wavelength and can be achieved using either a white light source, or the monochromatic calibration data used for the wavelength calibration. The resulting flat weight multiplies into the spectral weight column of the HDF5 table to merge with the linearity weight for each photon.

\section{Astrometric Calibration}

This is an implementation of a standard astrometric calibration to relate $(\mathrm{x}, \mathrm{y})$ pixel coordinates on the MKID array to sky coordinates using a linear mapping with rotation.

\section{Spectral Calibration}

This calibration accounts for the total QE of the detector and system as a function of wavelength. A spectrum is taken of a spectral standard with MEC and is compared to a calibrated spectrum of the same object. The resulting correction multiplies into the spectral weight column of the HDF5 table to merge with the flat weight and linearity weight for each photon.

\section{Output Generation}

There are a variety of output file formats that the MKID Pipeline can generate. In addition to FITS files which are compatible with more standard post-processing techniques, photon lists can be output directly to perform more specialized techniques that utilize the photon-counting ability of MKIDs.

\subsubsection{Analysis of Calibrated Data}

After the photon lists have been calibrated we can begin the analysis of astrophysical data produced by MEC. The easiest to understand procedure is to bin the photon list in time and wavelength to create images after which 
classical astronomy post-processing techniques can be used.

Figure 12 shows the five star system Theta ${ }^{1}$ Orionis B taken in y-band by MEC at Subaru Telescope on Jan. 12,2019 . B1, behind the coronagraph, is a spectroscopic eclipsing binary with nominal magnitude of $\mathrm{V}=7.96$. B2 and B3 are resolved by Subaru Telescope's $8.2 \mathrm{~m}$ mirror in conjunction with the $\mathrm{AO}$ correction provided by SCExAO (Jovanovic et al. 2015). The four astrogrid speckles artificially created by the DM are present in a square centered about B1, behind the coronagraph. These speckles are about 5.5 magnitudes fainter than B1. B4 is 4.98 magnitudes fainter than B1 in $\mathrm{H}$-band (Close et al. 2013). It is important to note that this observation was taken before the re-coating of the secondary mirror at Subaru which occurred on November 7th, 2019. The throughput hit taken before the mirror re-coating, coupled with the already dim guide star, B1, leads to a worse AO correction than is possible either now on the same system, or on a similar system with a brighter guide star.

The image uses data from 25 different $30 \mathrm{~s}$ observations located at different positions on-sky collected in a dither. We use a drizzling code to place the list of observed photons in a virtual pixel grid. that correctly takes into account the translation from the dither position as well as sky rotation as observed from the Nasmyth port. This helps to account for the lack of full array coverage due to dead feedlines and pixels to generate a complete image.

It is also possible to use the data directly without any binning in the calibrated photon list for more complex analysis, like the stochastic speckle discrimination technique outlined in Walter et al. (2019).

\section{MEC ON-SKY PERFORMANCE}

MEC underwent a major upgrade in late 2019 where we replaced internal microwave wiring that was degrading resonator performance due to crosstalk and excess noise (Smith et al. 2020). The results below reflect the performance after the upgrade. Due to fabrication problems we have also not yet been able to improve on the engineering grade MKID array in MEC, although we hope to install a science grade MKID array with all feedlines functional and higher yield in 2020. Luckily, most of the exoplanets of interest fit within the smaller field of view of the engineering grade array, although the full array will feature a 1.4" x 1.5" field of view to improve observations of disks.

\subsection{Yield}

Yield, or the number of functioning pixels compared to the total number of possible pixels, can be complex in an MKID array as every pixel is unique and can be compromised in a number of ways. For example, a spec of dust landing on a pixel during fabrication can short out a resonator, or merely move it to an unpredictable

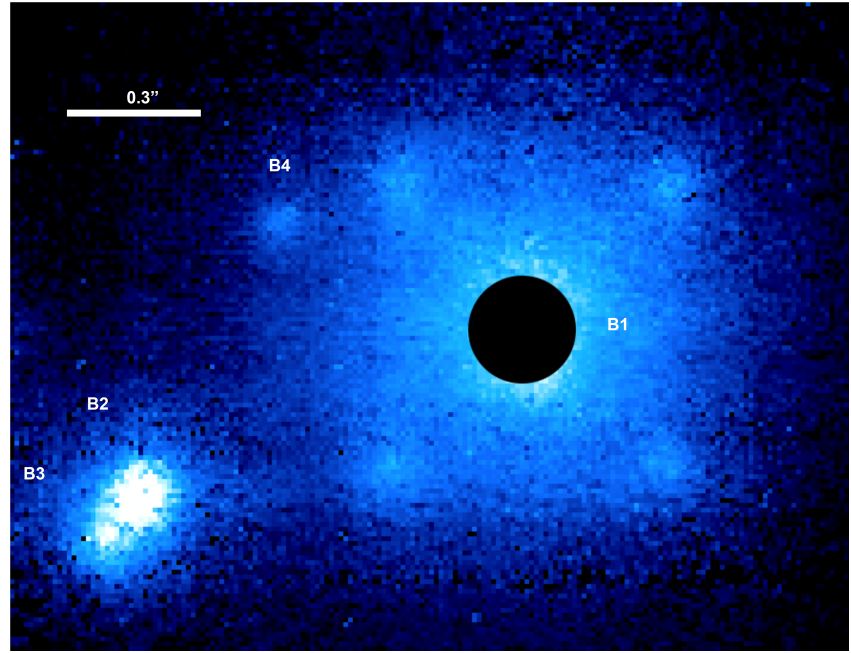

Figure 12. y-band image of Theta ${ }^{1}$ Orionis B taken by MEC at the Subaru Telescope on Jan. 12, 2019. A dither sequence of $2530 \mathrm{~s}$ images are stacked and then smoothed by a Gaussian filter. The black circle indicates the location of the coronagraph on B1. The four astrogrid speckles artificially created by the DM are present in a square centered about, and about 5.5 magnitudes fainter than, B1. B4 is 4.98 magnitudes fainter in $\mathrm{H}$ band than $\mathrm{B} 1$. The four artificial speckles are for astrometric and photometric calibration.

frequency. Non-uniformities in film thickness or composition can move resonators around in frequency, causing frequency overlaps that can render at least one of the pixels unable to be read out. Even worse, fabrication problems such as shorts due to photoresist bubbles can knock out entire feedlines, as seen in feedlines $2-4$ in the MEC engineering array.

The engineering MEC array pixel map is shown in Figure 13. For the fully working feedlines (6-10), $75 \%$ of pixels are photosensitive with $61.4 \%$ having a good wavelength calibration solution. When only looking at the best part of the detector (the "sweet spot"), between 20 and 60 in the y direction, these numbers increase to $82.1 \%$ and $74.3 \%$, respectively. The bad section in the center, between 60 and 80 in the y direction, is likely performing poorly as the device surface impedance was slightly higher than desired, so these resonators are lower frequency than designed (starting at 3.5 vs 3.9 $\mathrm{GHz}$ ), causing some issues with resonator coupling to the feedline and HEMT gain.

Simulations with the MEDIS package (Dodkins et al. 2020) have been performed to estimate the impact of dead pixels on the raw contrast (before post processing with ADI/SDI/SSD/etc.) for exoplanet direct imaging. These simulations show that the raw contrast will be roughly two times worse with observed yield in the sweet spot compared with perfect yield, as shown in Figure 14.

\subsection{Spectral Resolution}




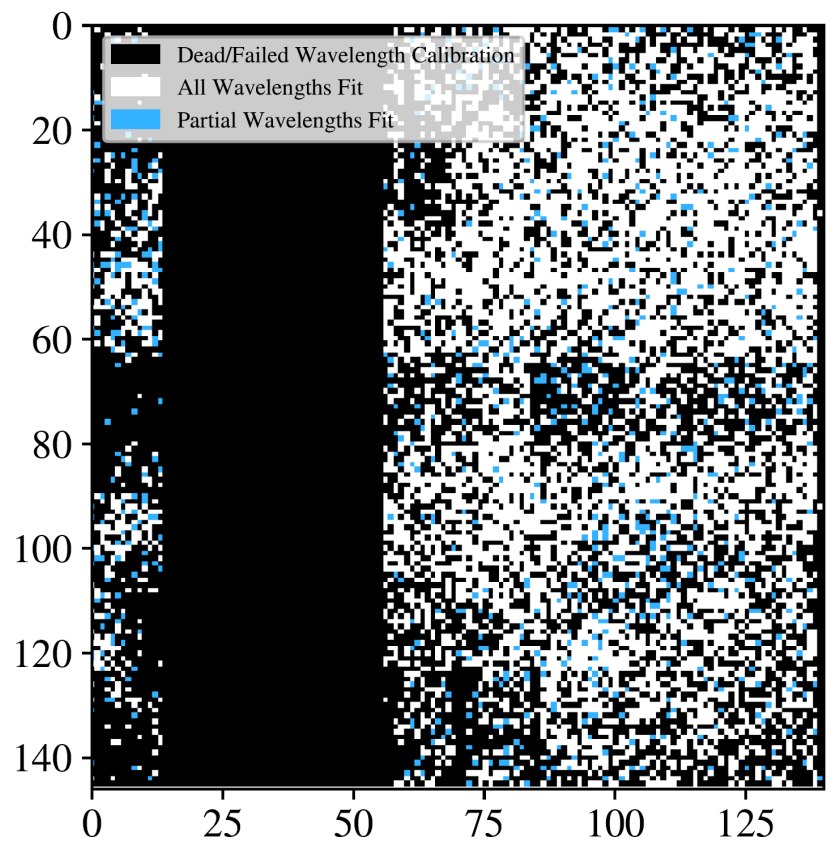

Figure 13. Wavelength calibration results for the engineering array in MEC. Fully wavelength calibrated pixels are shown in white, pixels calibrated using a subset of the available wavelengths are shown in blue, and pixels that had no wavelength calibration solution are shown in black.

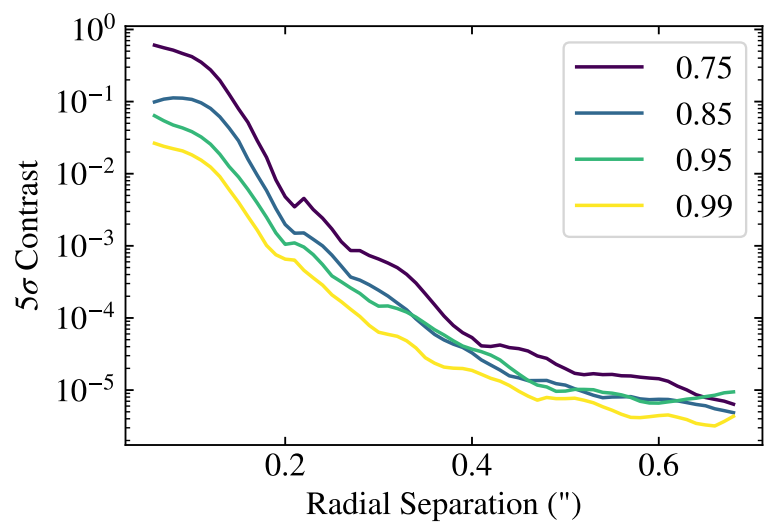

Figure 14. Simulation with the MEDIS package performed to evaluate the impact of dead pixels on the raw contrast of MEC. The four different lines represent the effect of varying pixel yield on the $5 \sigma$ contrast.

The spectral resolution of an MKID is a complex tradeoff between material, pixel geometry, and readout technique. The spectral resolution of the MKIDs used in MEC are explored in great detail in Zobrist et al. (2019). However, this data was taken under ideal circumstances in a dilution refrigerator in our lab, not in an ADR at the top of a mountain on an electrically noisy Naysmth
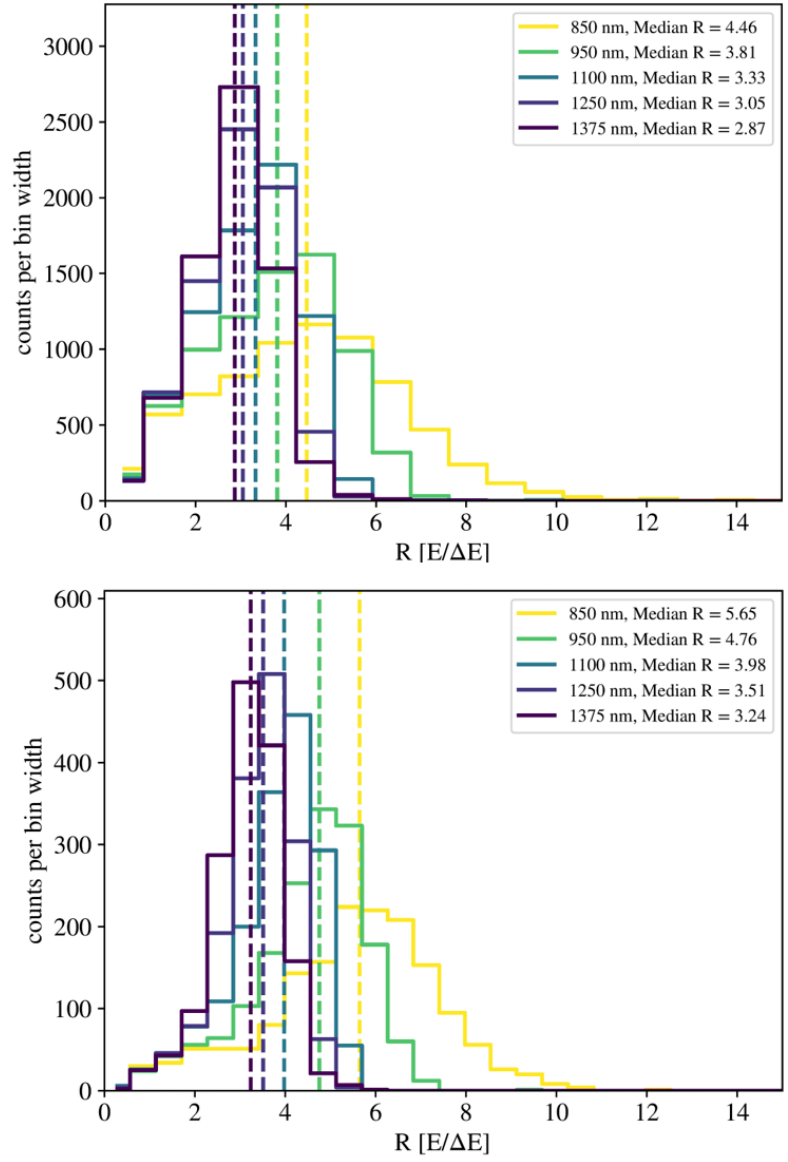

Figure 15. MEC spectral resolution for the whole array (top) and in the sweet spot (bottom). Note the differing vertical axes scales due to the smaller number of probed pixels in the best part of the array.

platform. Figure 15 shows the actual measured spectral resolution histograms of all the pixels in MEC, while Figure 16 shows a map of spectral resolution at a single wavelength.

A hand selected MKID pixel on a MEC-style PtSi array measured with a HEMT in the lab have a spectral resolution of around $\mathrm{R}=7.5$ at $980 \mathrm{~nm}$, while in MEC the median pixel in the good region of the array has $\mathrm{R}=5$ at $980 \mathrm{~nm}$. This degradation is likely related to several factors, including:

- Excess noise in the readout caused primarily by loss of signal in the high density microwave cables (Smith et al. 2020) that bring the signals to the HEMT.

- Intermodulation products causing unwanted tones that interfere with the pulse readout.

- Many pixels are not ideal due to fabrication errors, such as frequency overlaps. 


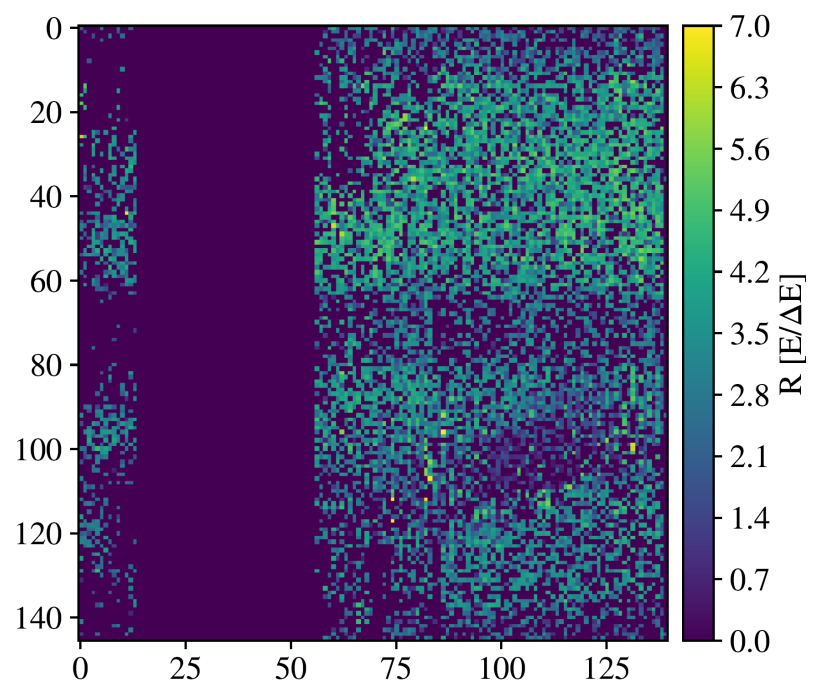

Figure 16. Map of energy resolution for MEC's engineering array at $1.1 \mu \mathrm{m}$

The readout itself does not appear to introduce noticeable degradation aside from the intermodulation products, which can be reduced through an optimization algorithm (Fruitwala Submitted).

\subsection{Throughput}

Throughput was calculated by comparing a spectrum of the G0 type star BD +172803 attained by MEC with a calibrated spectrum of the same target. This calibrated spectrum was generated by taking a G0 type stellar spectrum from the PHEONIX library and normalizing it to match the reported $\mathrm{J}$ and $\mathrm{H}$ band flux for this object (Husser et al. 2013; Cutri et al. 2003). In order to compare the two spectra, both were binned in $0.05 \mathrm{eV}$ bins which over samples the median MKID energy resolution by roughly a factor of 4 .

Dividing this binned MEC spectrum by the binned calibrated spectrum should yield the total system throughput as a function of wavelength, but there are some additional factors that must first be taken into consideration. During this observation, a 90/10 beamsplitter upstream of the MEC optics in SCExAO was used which directed only $10 \%$ of the total available light to MEC. Additionally, since this target has a J band magnitude of 6.6 and an $\mathrm{H}$ band magnitude of 6.4 , MEC's 2.5 OD filter had to be used so as to not saturate the array. This filter has not been measured as a function of wavelength for MEC and so represents a source of uncertainty in this calculation. Finally, the measured Strehl during this observation was found using the C-RED 2 IR camera in SCExAO to be $78 \%$ in $\mathrm{H}$ band which must also be accounted for since a tight aperture was used so as to only encompass the core of the PSF. Since the Strehl is expected to be worse at shorter wavelengths,

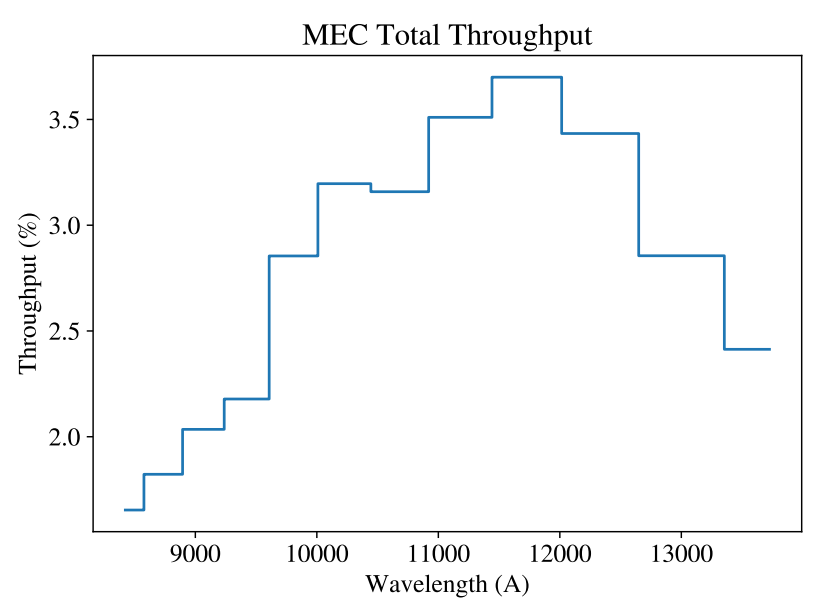

Figure 17. MEC throughput, from the top of the atmosphere to registered photons in the MKID detector, as a function of wavelength

using this measured $\mathrm{H}$ band Strehl of $78 \%$ represents a lower limit on the $\mathrm{J}$ band throughput.

Taking all of these factors into consideration, the resulting average $\mathrm{J}$ band throughput of the total optical system is $3.3 \pm 0.1 \%$, see Figure 17 . This is roughly consistent with what we expect if the total throughput of the atmosphere, telescope, AO188, and SCExAO are roughly $20 \%$. It is possible slight misalignment of the microlens is reducing detector quantum efficiency, so careful measurements of the science grade array $\mathrm{QE}$ will be performed before installation to allow modelling of the full system thoughput to compare with on-sky measurements.

\section{FUTURE PLANS}

\subsection{Upgrades to $M E C$}

Future hardware upgrades include a new antireflection coated science grade array which will roughly double the quantum efficiency and include fabrication process improvements that should greatly improve pixel yield. The MKID Pipeline will also be undergoing continual improvements which will allow us to more accurately determine the energy of each incident photon and to better remove false counts. For additional details on how future array improvements will affect the performance of MEC, see Dodkins et al. (2020).

\subsection{Wavefront Sensing and Control-Real-Time Integration with SCExAO}

MEC has already done on-sky speckle nulling using a code derived from Bottom et al. (2016) and demonstrated significant suppression of the quasi-static speckle halo which will be published in a future paper. This is the first step towards making MEC both a science camera and an effective focal plane wavefront sensor. Development is proceeding to speed speckle nulling up 


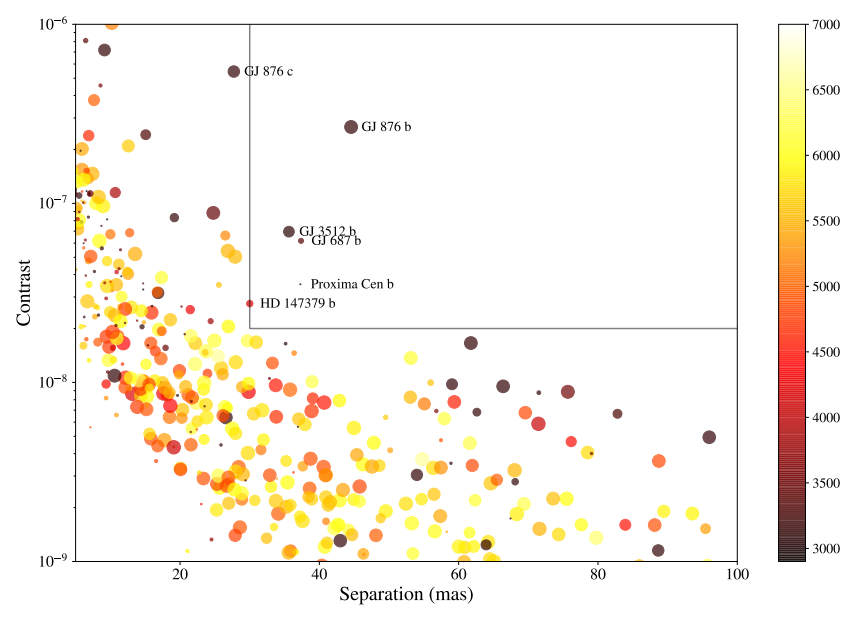

Figure 18. Angular separation vs. contrast for known exoplanets viewable with MEC/SCExAO and it's companion instrument DARKNESS (Meeker et al. 2018b) on MagAO-X. The size of the point represents the radius of the planet, and the color of the point represents the $\mathrm{T}_{\text {eff }}$ of the host star. The horizontal line at $2 \times 10^{-8}$ and the vertical line at 30 mas are meant to guide the eye to the most promising targets, which are labelled with their common names. Of these targets, GJ $876 \mathrm{~b}$ is by far the most promising.

to allow suppression of not just quasi-static speckles, but fast atmospheric speckles, with expected full probing and cancellation cycles occurring at frequencies of at least $200 \mathrm{~Hz}$. The implementation of more advanced coherent differential imaging (CDI) probing techniques (Matthews et al. 2017) are also being developed for use both in post-processing and in real time.

In the long term, a fully optimized real time control package using the Frazin algorithm (Frazin 2016, 2018) or similar predictive control (Males \& Guyon 2018) and sensor fusion approaches could help MEC use all available information simultaneously to approach fundamental photon noise limits and enable detection and characterization of smaller planets closer to their parent star. This technology will allow the next generation of instruments on 30-m class telescopes to potentially detect and characterize rocky planets in the habitable zones of nearby M-dwarfs.

\subsection{Science Goals}

MEC was designed with the goal of enabling imaging of exoplanets in reflected light. Figure 18 shows all currently known planets with their separation plotted against the expected contrast ratio. With MEC we hope to reach final contrasts of at $10^{-7}$ at $2 \lambda / D$ after post-processing, enabling the imaging of at least GJ $876 \mathrm{~b}$. This contrast limit is theoretically achievable by MEC given the inner working angle of the instrument and assuming we are photon noise dominated. Future papers, as mentioned above, will detail the use of both high speed realtime and post-processing techniques to reach this noise limit and ultimately enhance the discovery reach of SCExAO.

In addition to reflected light planets, MEC is also a powerful tool for the discovery and characterization of young giant planets still glowing from the heat of their formation. The shorter wavelengths MEC probes allows for superior angular resolution, and the high final contrast at small inner working angles, due to focal plane wavefront sensing and novel post-processing, should allow us to probe new parameter space.

\section{ACKNOWLEDGEMENTS}

Sarah Steiger and Neelay Fruitwala are supported by a grant from the Heising-Simons Foundation. Kristina K. Davis is supported by the NSF Astronomy and Astrophysics Postdoctoral Fellowship program under award number 1801983. Jennifer P. Smith and Nicholas Zobrist are both supported by a NASA Space Technology Research Fellowship. Isabel Lipartito is supported by the National Science Foundation Graduate Research Fellowship under grant number 1650114. Frantz Martinache is funded by the European Research Council (ERC) under the European Union's Horizon 2020 research and innovation program (grant agreement CoG - 683029).

The development of SCExAO was supported by the Japan Society for the Promotion of Science (Grant-inAid for Research \#23340051, \#26220704, \#23103002, \#19H00703 \& \#19H00695), the Astrobiology Center of the National Institutes of Natural Sciences, Japan, the Mt. Cuba Foundation and the director's contingency fund at Subaru Telescope. The authors wish to recognize and acknowledge the very significant cultural role and reverence that the summit of Maunakea has always had within the indigenous Hawaiian community, and are most fortunate to have the opportunity to conduct observations from this mountain.

\section{REFERENCES}

Beuzit, J.-L., Vigan, A., Mouillet, D., et al. 2019, Astronomy \& Astrophysics, 631, A155

Bottom, M., Femenia, B., Huby, E., et al. 2016, in Proceedings of the SPIE, California Institute of Technology (United States) (International Society for Optics and Photonics), 990955
Bowler, B. P. 2016, PASP, 128, 102001

Carbillet, M., Bendjoya, P., Abe, L., et al. 2011, Experimental Astronomy, 30, 39, doi: $10.1007 /$ s10686-011-9219-4

Close, L. M., Males, J. R., Morzinski, K., et al. 2013, ApJ, 774,94 
Crepp, J. R., Pueyo, L., Brenner, D., et al. 2011, ApJ, 729, 132

Currie, T., Daemgen, S., Debes, J., et al. 2014, The Astrophysical Journal Letters, 780, L30

Cutri, R., Skrutskie, M., Van Dyk, S., et al. 2003, tmc

Day, P., Leduc, H., A Mazin, B., Vayonakis, A., \& Zmuidzinas, J. 2003, Nature, 425, 817, doi: 10.1038/nature02037

Dodkins, R., Mahashabde, S., O'Brien, K., et al. 2018, Astronomy and Computing, 23, 60

Dodkins, R. H., Davis, K. K., Lewis, B., et al. 2020, Publications of the Astronomical Society of the Pacific, 132, 104503

Frazin, R. A. 2016, Journal of the Optical Society of America A, 33, 712

—. 2018, Journal of the Optical Society of America A, 35, 594

Fruitwala, N. Submitted, AIP Review of Scientific Instruments

Gerard, B. L., Marois, C., Currie, T., et al. 2019, ApJ, 158, 36

Gomez Gonzalez, C. A., Absil, O., Absil, P.-A., et al. 2016, A\&A, 589, A54

Guyon, O. 2005, ApJ, 629, 592

Hodapp, K. W., Suzuki, R., Tamura, M., et al. 2008, in Ground-based and Airborne Instrumentation for Astronomy II, Vol. 7014, International Society for Optics and Photonics, 701419

Husser, T.-O., Wende-von Berg, S., Dreizler, S., et al. 2013, Astronomy \& Astrophysics, 553, A6

Jovanovic, N., Martinache, F., Guyon, O., et al. 2015, PASP, 127, 890

Jovanovic, N., Delorme, J.-R., Bond, C. Z., et al. 2019, arXiv preprint arXiv:1909.04541

Keppler, M., Benisty, M., Müller, A., et al. 2018, A\&A, 617, A44

Kuzuhara, M., Tamura, M., Kudo, T., et al. 2013, ApJ, 774,11

Lafrenière, D., Marois, C., Doyon, R., Nadeau, D., \& Artigau, É. 2007, ApJ, 660, 770

Lagrange, A. M., Bonnefoy, M., Chauvin, G., et al. 2010, Science, 329, 57

Lewis, B., \& Oppenheimer, R. 2017, Columbia Undergraduate Science Journal, 11

Lozi, J., Guyon, O., Jovanovic, N., et al. 2018, Journal of Astronomical Telescopes, Instruments, and Systems, 4, 1

Macintosh, B., Graham, J. R., Ingraham, P., et al. 2014, Proceedings of the National Academy of Sciences, 111, 12661, doi: 10.1073/pnas.1304215111
Macintosh, B., Graham, J. R., Barman, T., et al. 2015, Science, 350, 64

Macintosh, B. A., Grahamand, J. R., Palmer, D. W., et al. 2008, SPIE, 7015, 7015

Males, J. R., \& Guyon, O. 2018, Journal of Astronomical Telescopes, 4, 019001

Males, J. R., Close, L. M., Miller, K., et al. 2018, SPIE, 10703

Marois, C., Correia, C., Galicher, R., et al. 2014, in SPIE, Vol. 9148, Adaptive Optics Systems IV, 91480U

Marois, C., Doyon, R., Racine, R., \& Nadeau, D. 2000, PASP, 112, 91

Marois, C., Lafrenière, D., Doyon, R., Macintosh, B., \& Nadeau, D. 2006, ApJ, 641, 556

Marois, C., Macintosh, B., Barman, T., et al. 2008, Science, 322,1348

Matthews, C. T., Crepp, J. R., Vasisht, G., \& Cady, E. 2017, Journal of Astronomical Telescopes, 3, 045001

Mawet, D., Shelton, C., Wallace, J., et al. 2014a, SPIE, 9143, 9143

Mawet, D., Milli, J., Wahhaj, Z., et al. 2014b, ApJ, 792, 97

Mawet, D., Wizinowich, P., Dekany, R., et al. 2016, SPIE, 9909, 9909

Mazin, B. A., Bumble, B., Meeker, S. R., et al. 2012, Opt. Express, 20, 1503

Mazin, B. A., Meeker, S. R., Strader, M. J., et al. 2013, PASP, 125, 1348

McHugh, S., Mazin, B. A., Serfass, B., et al. 2012, Review of Scientific Instruments, 83, 044702, doi: 10.1063/1.3700812

Meeker, S. R., Mazin, B. A., Walter, A. B., et al. 2018a, PASP, 130

—. 2018b, Publications of the Astronomical Society of the Pacific, 130, 065001

Racine, R., Walker, G. A. H., Nadeau, D., Doyon, R., \& Marois, C. 1999, PASP, 111, 587

Smith, J. P., Mazin, B. A., Walter, A. B., et al. 2020, IEEE Transactions on Applied Superconductivity, 31, 1

Soummer, R., Pueyo, L., \& Larkin, J. 2012, ApJ, 755, L28

Sparks, W. B., \& Ford, H. C. 2002, ApJ, 578, 543

Strader, M. 2016, PhD thesis, University of California Santa Barbara

Szypryt, P., Meeker, S. R., Coiffard, G., et al. 2017, Opt. Express, 25, 25894

van Eyken, J. C., Strader, M. J., Walter, A. B., et al. 2015, ApJS, 219, 14

Walter, A. B., Bockstiegel, C., Brandt, T. D., \& Mazin, B. A. 2019, Publications of the Astronomy Society of the Pacific, 131, 114506 
Zobrist, N., Eom, B. H., Day, P., et al. 2019, Applied Physics Letters, 115, 042601 